\title{
Langerhans Cell Histiocytosis with Atypical Intervertebral Disc and Sacroiliac Joint Involvement Mimicking Osteoarticular Tuberculosis in an Adult
}

\author{
Zeynep Maraş Özdemir ${ }^{1}$, Ayşegül Sağır Kahraman ${ }^{1}$, Cemile Ayșe Görmeli ${ }^{1}$, Reşit Sevimli² ${ }^{2}$ Nusret Akpolat ${ }^{3}$
}

\author{
${ }^{1}$ Department of Radiology, İnönü University School of Medicine, Malatya, Turkey \\ ${ }^{2}$ Department of Orthopedics and Traumatology, İnönü University School of Medicine, Malatya, Turkey \\ ${ }^{3}$ Department of Pathology, İnönü University School of Medicine, Malatya, Turkey
}

Background: Langerhans cell histiocytosis ( $\mathrm{LCH})$, typically found in children, is a rare single or multisystem disorder with a wide range of clinical and radiological manifestations. Unusual presentations of LCH are occasionally encountered and it may be difficult to distinguish LCH from an infection or a benign or malignant tumor.

Results: A 35-year-old female presented with pain in her back and left buttock, malaise, and weight loss, with a duration of several months. Her laboratory test results were within the normal ranges except for the levels of acute phase reactants, which were elevated. Magnetic resonance imaging and computed tomography revealed a unilateral destructive sacroiliac lesion, and multiple vertebral lesions with adjacent discal involvement and extensive soft tissue extensions. She was initially misdiagnosed with multifocal osteoarticular tuberculosis. An open biopsy and joint curettage was performed. Histopathological examination showed that she had LCH.

Conclusion: To the best of our knowledge, this is the first case of $\mathrm{LCH}$ associated with a destructive unilateral sacroiliac lesion, discal involvement, and involvement of the adjacent vertebrae, in an adult patient; the LCH mimicked osteoarticular tuberculosis. Disease onset in adulthood is rare, and this can potentially delay diagnosis. Familiarity with the imaging features of unusual LCH manifestations is necessary to ensure accurate diagnosis and appropriate treatment.

Keywords: Langerhans cell histiocytosis, sacroiliac joint, intervertebral disc, tuberculosis, osteoarticular
Langerhans cell histiocytosis (LCH), which most commonly occurs in children, is a rare disorder of unknown etiology characterized by uncontrolled monoclonal proliferation of Langerhans cells (1). Bone and skin lesions are the most common manifestations of disease in children (2); in adults, the most commonly affected sites are bone and the lungs (3). A few articles have described unusual skeletal and soft tissue manifestations of LCH (4-8). Becoming familiar with the unusual radiological findings, which can mimic an infection or benign or malignant tumor is important because the treat- ment options differ greatly from those appropriate for other conditions.

Here, we report an interesting case of extensive soft tissue infiltration of the spinal column and unilateral sacroiliac joint, mimicking tuberculous spondylodiscitis and sacroiliitis, in a 35-year-old woman with multisystem LCH diagnosed via histopathological examination. To the best of our knowledge, neither intervertebral disc nor sacroiliac joint infiltration by $\mathrm{LCH}$ has been previously described. 


\section{CASE PRESENTATION}

A 35-year-old female presented with pain in her back and left buttock, malaise, and weight loss. Her complaints were of several months duration. Her family and medical history were unremarkable. Upon physical examination, her body temperature was normal. No motor weakness or sensory deficit in the lower extremities were found. The hip range-of-motion was normal, although excessive motion (in all directions) of the left hip elicited pain. The left iliac wing and the spinous processes of the lower thoracic and lumbar vertebrae were also painful when pressure was applied. Blood data were in the normal range, except that the acute phase reactants were elevated (the erythrocyte sedimentation rate was $44 \mathrm{~mm} / \mathrm{h}$ and the C-reactive protein level $7.86 \mathrm{mg} / \mathrm{dL}$ ). The Brucella agglutination test was negative.

Magnetic resonance imaging (MRI) (Avanto, Siemens; Erlangen, Germany) revealed an abscess-like lesion, with irregular peripheral rim-like enhancement, in the left sacroiliac joint space (Figure $1 \mathrm{a}-\mathrm{c}$ ). The joint space was enlarged because of destruction of the articular surface. Computed tomography (CT) (Aquilion 64, Toshiba; California, USA) of the pelvis revealed significant destruction and irregularities of the periarticular bones, and sequestra within the joint (Figure 1e). MRI also revealed edema-like signal changes and diffuse contrast enhancement in the periarticular soft tissue, which spread anteriorly to the presacral region and left piriformis muscle, medially to the left sacral foramina, laterally to the gluteal muscle and deep subcutaneous connective tissue, and posteriorly and posteromedially to the left paraspinal muscle and deep subcutaneous connective tissue (Figure 1c,d). In addition, MRI of the lumbar spine revealed edema-like signal changes and contrast enhancement in the adjacent end-plates of L4 and the posterior T9-T10-T11 vertebrae, with extension of the adjacent intervertebral disc spaces and the foramina. Similar signal changes were evident in the isolated T7-8 disc space, without involvement of the adjacent vertebrae. There was also involvement of the spinous process of $\mathrm{T} 8$ associated with adjacent soft tissue signal abnormalities. All MRI signal abnormalities evident in the vertebral column spread to the paraspinal and perineural soft tissues in an infiltrative pattern (Figure 2). In addition, many of the para-aortic and para-iliac lymph nodes were enlarged, and some were conglomerated on MRI (Figure 2). Thoracic CT, preformed preoperatively, revealed thin-walled air-filled cysts, mostly $<10 \mathrm{~mm}$ in diameter, and multiple small $(<5 \mathrm{~mm})$ bilateral nodules in all lung
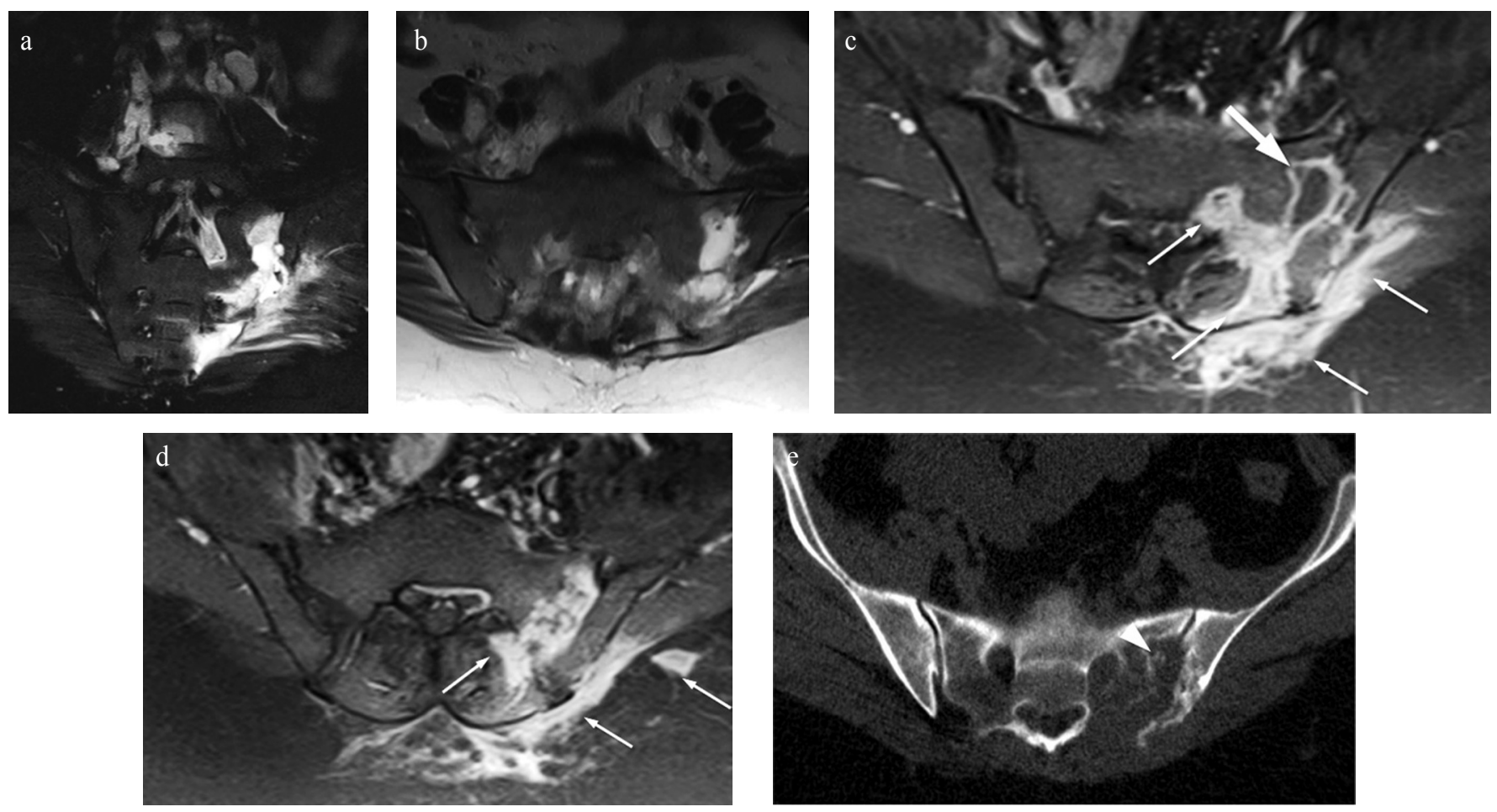

FIG. 1. a-e. Coronal fat-saturated T2-weighted (a), axial T2-weighted (b) MR images show extensive edema-like and fluid-like signal changes within the left sacroiliac joint space and periarticular soft tissue with enlarged joint space. Axial intravenous contrast-enhanced fat-saturated T1-weighted (c,d) MR images show irregular peripheral rim-like enhancement within the joint space (thick arrow, c) which resembles an abscess formation. MR images also show extensive edema like-signal changes and diffuse contrast enhancement in the periarticular soft tissue, which spread to the sacral foramina, paraspinal and gluteal muscles and subcutaneous fat tissue (thin arrows, c,d). CT images of the pelvis (e) show significant destruction of the periarticular bones with sequestra into the joint space (arrowhead). Note also intervertebral disc and paravertebral tissue involvement in a. 

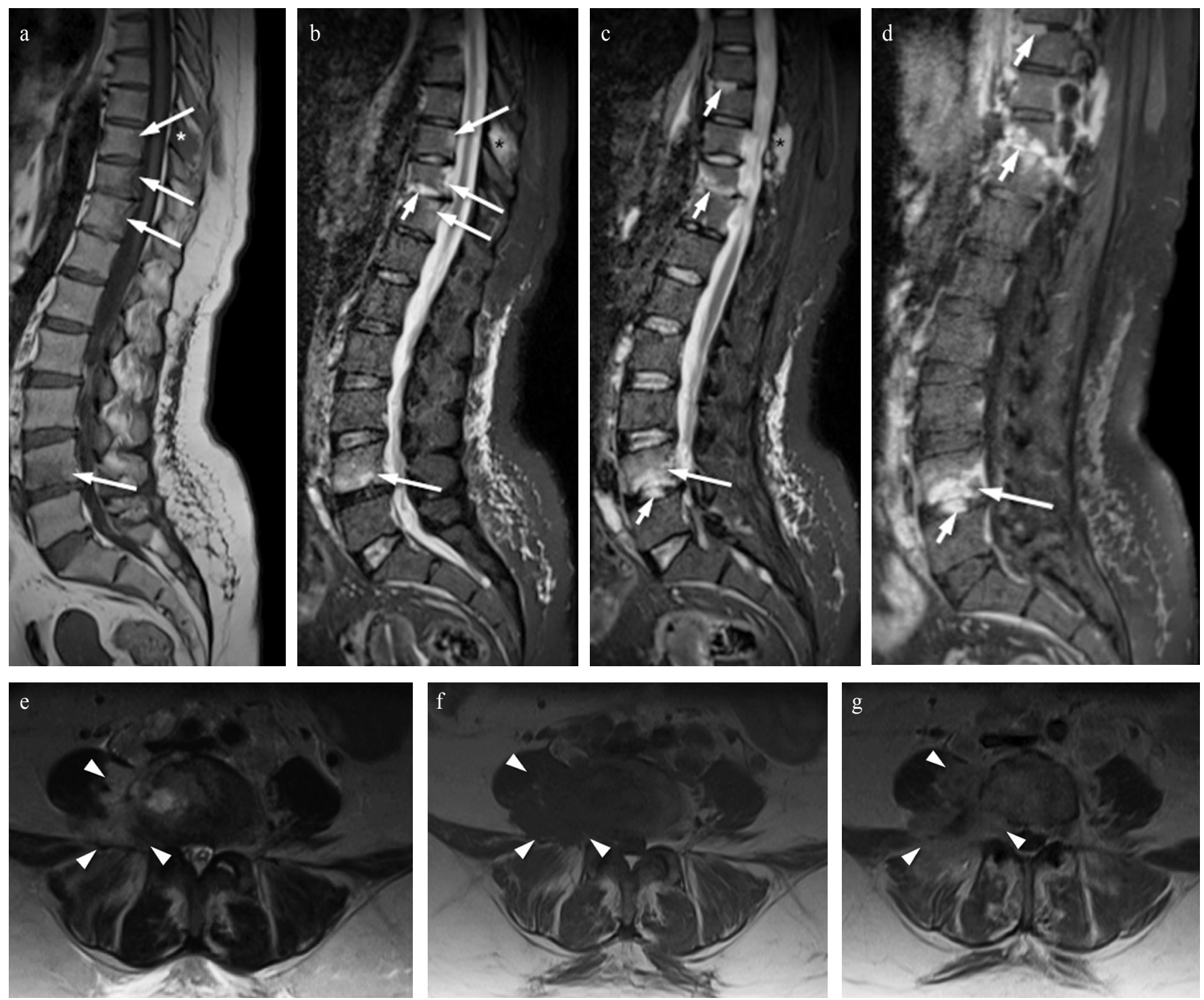

FIG. 2. a-g. Sagittal T1-weighted (a), sagittal short-tau inversion recovery (STIR) (b, c) and sagittal contrast-enhanced fat-saturated T1-weighted (d) MR images of the spine show vertebral (long arrows) and intervertebral disc (short arrows) involvement at multiple levels. The involvement of the spinous process of T8 is also noted (asterisks). Disc lesion is isolated at the T7-8 level while disc involvement is associated with adjacent vertebral end-plate lesion at the level of L4-5 and T10-11 (short arrows). Axial T2-wieghted (e), T1-weighted (f) and contrast-enhanced T1-weighted (g) MR images show perineural and paravertebral soft tissue extension (arrowheads). Note also the involvement of several para-aortic lymph nodes in c, d, e.

zones (predominantly the lower zones) with pleural effusion and mediastinal lymphadenopathy.

The clinical and radiological features led us to initially diagnose osteoarticular tuberculosis with unilateral sacroiliac joint and multiple spinal involvement, but the operative findings were incompatible with septic arthritis of the sacroiliac joint. An open biopsy and joint curettage were performed, using a posterior approach. Histopathological evaluation identified $\mathrm{LCH}$, and CD1a and S100 were detected immunohistochemically (Figure 3). Written informed consent was obtained from the patient for this report.

\section{DISCUSSION}

Langerhans cell histiocytosis, also known as histiocytosis $\mathrm{X}$, is a rare disorder characterized by abnormal proliferation of the Langerhans cells and the accumulation of such cells in various tissues and systems (1). The features of the disease are confusing and remain poorly described in adults because most patients are young children (1-3). Here, we describe a case of multisystem LCH affecting several intervertebral disc spaces, with unilateral sacroiliac joint involvement (thus mimicking osteoarticular tuberculosis). This form of 

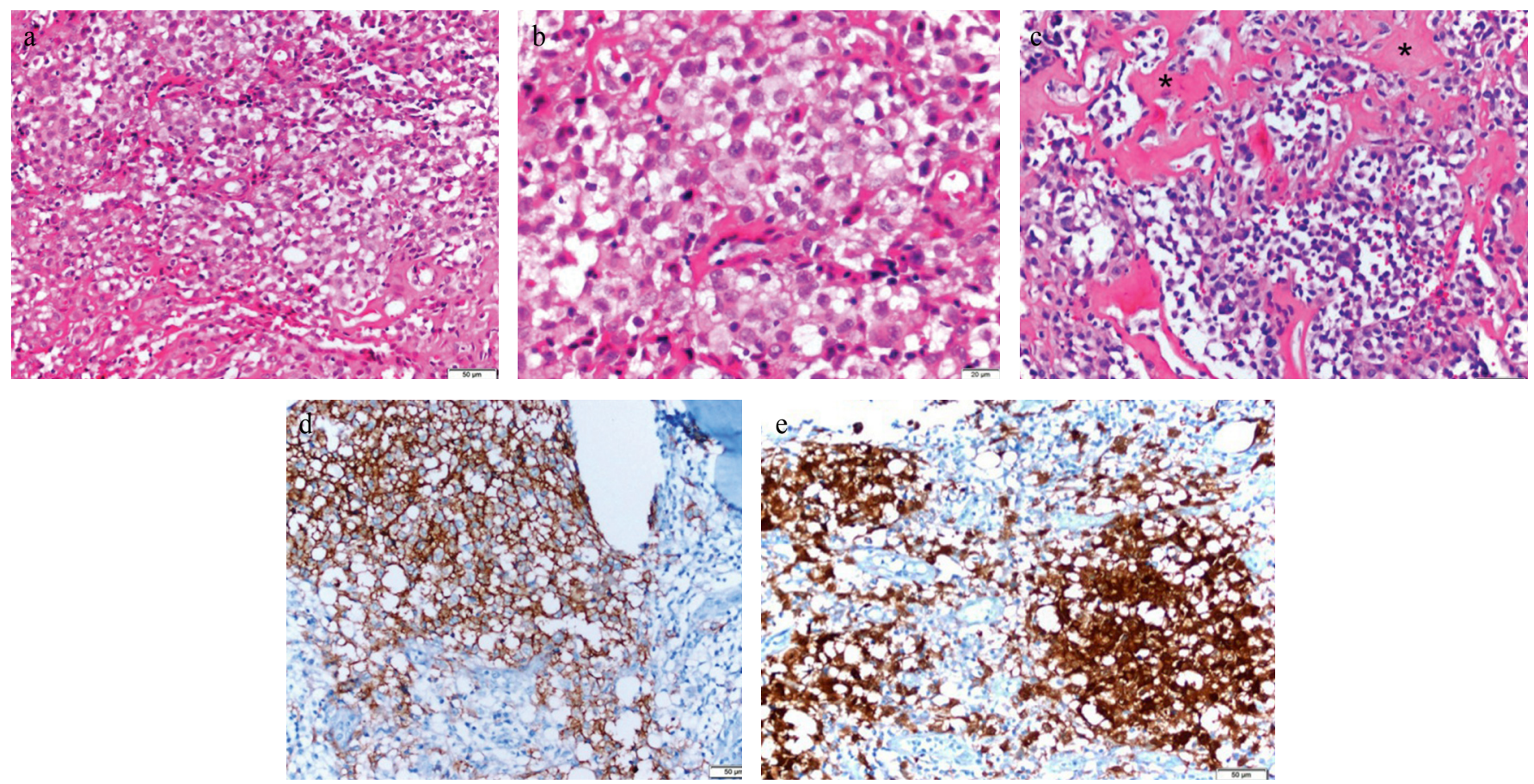

FIG. 3. a-e. Sacroiliac joint biopsy specimen shows infiltration of Langerhans cell histiocytosis with histiocyte-like cells and eosinophils (a, hematoxylin and eosin, original magnification, x400). High magnification (b) reveals characteristic folded nuclei of Langerhans cells. Note also the infiltration of the bone (asterisks) by neoplastic cells on c (hematoxylin and eosin, original magnification, $\mathrm{x} 200$ ). Immunohistochemical analysis of the specimen shows that tumor cells are positive for CD1a (d, immunoperoxidase x200) and S100 (e, immunperoxidase x200).

LCH presentation has not previously been reported in either children or adults.

Unusual manifestations of $\mathrm{LCH}$ in terms of bone and soft tissue involvement have been described in the literature (4$8)$. These include multifocal soft tissue lesions with or without secondary bone involvement (4-7), and unusual skeletal manifestations (lesions of the posterior vertebral arc, dural extensions of vertebral lesions, fluid-fluid lesions, etc.) (8). Osseous involvement in LCH typically involves the flat bones, predominantly those of the skull (1). Spinal lesions occur in the single vertebral body without neural arch and paravertebral/epidural soft tissue extension in most cases $(1,9)$. However, rare spinal presentations include posterior arch extensions, paravertebral and epidural soft tissue masses, and neurological manifestations, as described in the literature (7-9). In advanced stages of disease, the vertebral body may collapse uniformly. This condition is termed vertebra plana, and is typically found in children. The intervertebral disc spaces are preserved, or appear to be slightly widened, even at this stage $(1,7,9)$. Our patient had an unusual pattern of spinal involvement, including posterior arch involvement with extreme paravertebral and perineural soft tissue extensions. Moreover, the adjacent endplates were affected, and disc involvement was apparent; neither of these findings has been previously reported in the literature.
A few studies on soft tissue LCH have appeared. LCH accompanied by soft tissue masses, with or without involvement of the adjacent bone, is infrequent, and usually develops in the lower extremities (4-7). Only one of the cited articles mentions joint involvement (of the atlantoaxial joint). However, this case report describes a large soft tissue mass at the nape of the neck, with destruction of the posterior element of the atlas and the axis, irrelevant of an arthritis-like condition (7). Our case presented with a unilateral destructive sacroiliac lesion. To the best of our knowledge, such joint involvement in $\mathrm{LCH}$ has not previously been reported. Infections, particularly tuberculosis, should always be considered in the differential diagnosis when a patient exhibits unilateral destructive sacroiliitis and soft tissue extensions (10). Moreover, a combination of vertebral and sacroiliac involvement suggests the possibility of multifocal osteoarticular tuberculosis, although such a combination of joint involvement in tuberculosis is a rare condition (10).

The clinical picture of our patient (insidious disease onset and progression, with nonspecific symptoms) and the imaging findings (including a unilateral severe destructive sacroiliac lesion with periarticular soft tissue extensions, involvement of the disc space and adjacent endplates, posterior arch abnormalities of multiple vertebral segments with widespread paravertebral and perineural soft tissue extensions) 
rendered it difficult to distinguish LCH from osteoarticular tuberculosis.

The importance of this case is threefold: i) the adult onset of the disease is much rarer and may potentially delay diagnosis, ii) extensive involvement of the unilateral sacroiliac joint may mimic that of infective sacroiliitis, especially secondary to tuberculosis, and iii) disc extension of the lesions may be observed, unusual for $\mathrm{LCH}$, which has never been reported to involve the disc spaces in the literature before. Familiarity with the imaging features of $\mathrm{LCH}$ and a high index of clinical suspicion are necessary to ensure correct diagnosis and appropriate treatment.

\section{Ethics Committee Approval: N/A.}

Informed Consent: Written informed consent was obtained from patient who participated in this study.

Peer-review: Externally peer-reviewed.

Author contributions: Concept - Z.M.Ö, A.S.K, C.A.G.; Design - Z.M.Ö, A.S.K.; Supervision - Z.M.Ö.; Resource - Z.M.Ö, R.S, N.A.; Materials - C.A.G, A.S.K.; Data Collection and/or Processing - Z.M.Ö., R.S., N.A.; Analysis and/or Interpretation - C.A.G, A.S.K., N.A.; Literature Search - Z.M.Ö, C.A.G; Writing - Z.M.Ö., A.S.K.; Critical Reviews - C.A.G., N.A.

Conflict of Interest: No conflict of interest was declared by the authors.

Financial Disclosure: The authors declared that this study has received no financial support.

\section{REFERENCES}

1. Stull MA, Kransdorf MJ, Devaney KO. Langerhans cell histiocytosis of bone. Radiographics 1992;12:801-23. [CrossRef]

2. Schmidt S, Eich G, Geoffray A, Hanquinet S, Waibel P, Wolf $\mathrm{R}$, et al. Extraosseous langerhans cell histiocytosis in children. Radiographics 2008;28:707-26. [CrossRef]

3. Arico M, Girschikofsky M, Genereau T, Klersy C, McClain K, Grois N, et al. Langerhans cell histiocytosis in adults. Report from the International Registry of the Histiocyte Society. Eur $J$ Cancer 2003;39:2341-8. [CrossRef]

4. Amini B, Kumar R, Wang WL. Soft tissue Langerhans cell histiocytosis with secondary bone involvement in extremities: evolution of lesions in two patients. Skeletal Radiol 2013;42:1301-9. [CrossRef]

5. Samet J, Weinstein J, Fayad LM. MRI and clinical features of Langerhans cell histiocytosis (LCH) in the pelvis and extremities: can LCH really look like anything? Skeletal Radiol 2016;45:607-13. [CrossRef]

6. Onal C, Oymak E, Reyhan M, Canpolat T, Ozyilkan O. Multifocal soft tissue Langerhans' cell histiocytosis treated with PETCT based conformal radiotherapy. Jpn J Radiol 2015;33:603-6. [CrossRef]

7. Mondal D, Julka PK, Jana M, Walia R, Chaudhuri T. Langerhans cell histiocytosis of atlantoaxial joint in a middle-aged man presenting with deafness as first symptom and soft-tissue mass at neck showing excellent response to radiotherapy alone: Report of an extremely rare and unusual clinical condition and review of literature. Ann Indian Acad Neurol 2014;17:429-32. [CrossRef]

8. Hindman BW, Thomas RD, Young LW, Yu L. Langerhans cell histiocytosis: unusual skeletal manifestations observed in thirtyfour cases. Skeletal Radiol 1998;27:177-81. [CrossRef]

9. Jiang L, Liu XG, Zhong WQ, Ma QJ, Wei F, Yuan HS, et al. Langerhans cell histiocytosis with multiple spinal involvement. Eur Spine J 2011;20:1961-9. [CrossRef]

10. Gelal F, Sabah D, Doğan R, Avci A. Multifocal skeletal tuberculosis involving the lumbar spine and a sacroiliac joint: MR imaging findings. Diagn Interv Radiol 2006;12:139-41. 